\title{
Avaliação Muscular Isocinética da Articulação do Ombro em Atletas da Seleção Brasileira de Voleibol Sub-19 e Sub-21 Masculino
}

\section{Isokinetic Muscular Assessment of the Shoulder Joint in Athletes From the Male Under-19 and Under-21 Brazilian Volleyball Teams}

Luciana De Michelis Mendonça' Natalia Franco Netto Bittencourt ${ }^{2}$ Marco Túlio Saldanha dos Anjos ${ }^{3}$ Anderson Aurélio da Silva ${ }^{4}$ Sérgio Teixeira Fonseca

1. Fisioterapeuta do Laboratório de Prevenção e Reabilitação de Lesões Esportivas (LAPREV - Centro de Excelência Esportiva - CENESP da Universidade Federal de Minas Gerais - UFMG). Fisioterapeuta da Seleção Brasileira de Voleibol Sub-19 Masculina. Professora do Instituto Mineiro de Acupuntura e Massagens (INCISA)

2. Fisioterapeuta do LAPREV CENESP/UFMG. Fisioterapeuta da Seleção Brasileira de Voleibol Sub-17 Feminina e do Minas Tênis Clube. 3. Fisioterapeuta do LAPREV CENESP/UFMG. Professor do Centro Universitário Newton Paiva.

4. Professor do Departamento de Fisioterapia da Escola de Educação

Física, Fisioterapia e Terapia

Ocupacional da Universidade

Federal de Minas Gerais.

Coordenador do LAPREV.

5. Professor Doutor do

Departamento de Fisioterapia

da Escola de Educação Física,

Fisioterapia e Terapia Ocupacional da Universidade Federal de Minas Gerais. Coordenador do LAPREV.

Endereço para correspondência: Rua Minas Novas 104/901 Bairro: Cruzeiro

Belo Horizonte- Minas Gerais

Cep: 30310-090

E-mail: lucianademichelis@yahoo.com.br

\section{RESUMO}

Introdução e objetivo: A musculatura do complexo do ombro possui um papel importante na produção de força e potência articular no voleibol, permitindo a realização do gesto esportivo de maneira coordenada. A documentação objetiva produzida pela avaliação isocinética auxilia no diagnóstico e na prevenção de lesões do complexo do ombro, além de ser uma ferramenta útil no prognóstico do retorno rápido e seguro para a prática esportiva do atleta lesionado. O objetivo deste estudo foi avaliar o desempenho muscular isocinético dos músculos rotadores laterais e mediais da articulação glenoumeral em atletas de voleibol da Seleção Brasileira Masculina sub-19 e sub-21. Métodos: O protocolo realizado durante o teste foi de cinco repetições máximas de rotação lateral e medial de ombro no modo concêntrico-concêntrico na velocidade de $60 \%$ s e 30 repetições na velocidade de $360 \%$ s. Análise de variância (ANOVA) mista foi utilizada para avaliar as variáveis dependentes: trabalho máximo e torque máximo normalizados pela massa corporal dos músculos rotadores laterais e mediais do ombro, razão agonista/antagonista nas duas velocidades e índice de fadiga muscular. Resultados: A análise dos dados demonstrou haver um desequilíbrio entre os rotadores laterais e mediais (razão agonista/antagonista) da articulação do ombro em atletas brasileiros de voleibol da categoria sub-21, revelando menor força relativa dos rotadores laterais. Conclusão: Foi possível identificar alterações em atletas de voleibol que, associadas a demandas específicas do esporte, podem levar à sintomatologia no complexo do ombro e/ou queda da performance.

Palavras-chave: torque, ombro, lesões esportivas.

\section{ABSTRACT}

Introduction and purpose: The shoulder complex muscles play an important role in force, power and endurance production in volleyball, allowing the accomplishment of movement in a coordinated manner. The objective written account produced by isokinetic evaluations assists in the diagnosis and prevention of shoulder complex injuries. In addition, it is a useful tool in promoting safe and fast return of the injured athlete to sports practice. The purpose of this study was to analyze the isokinetic muscle performance of the lateral and medial rotators of the glenohumeral joint in Brazilian male volleyball teams (under-19 and under-21). Methods: The protocol used during the test included five maximum repetitions of shoulder lateral and medial rotation in concentric-concentric mode at $60 \%$ and 30 repetitions at $360 \%$ s. Analysis of variance was used to evaluate the dependent variables: maximum work and maximum torque of shoulder lateral and medial rotators normalized by body mass, antagonist/agonist ratio at the two speeds and muscular fatigue index. Results: Data analysis demonstrated an imbalance between the shoulder lateral and medial rotators (antagonist/agonist ratio) in the under-21 category of the Brazilian volleyball athletes, demonstrating relative weakness of the lateral rotators. Conclusion: It was possible to identify changes in volleyball players, which added to specific demands of the sport, can lead to symptoms in the shoulder complex and / or decrease of performance.

Keywords: shoulder, torque, athletic injuries. 


\section{INTRODUÇÃO}

O voleibol demanda dos membros superiores movimentos de alta velocidade em cadeia cinética aberta. Nesse sentido, o movimento de cortada é um elemento determinante para se atingir um alto nível nesse esporte ${ }^{(1)}$ Um atleta de alto rendimento do voleibol que treina de 16 a 20 horas por semana chega a realizar cerca de 40.000 movimentos de cortada por ano. Devido a essa demanda e à importância do ataque no sistema tático do voleibol, tem sido dada grande ênfase ao treinamento do gesto especifico da cortada ${ }^{(1,2)}$.

O movimento de cortada pode ser dividido nas fases de aproximação, impulsão (contato no solo antes da saída), saída, voo (antes do contato com a bola), batida, aterrissagem e recuperação(2). A primeira fase possibilita ao atleta ganhar um momentum horizontal, que será convertido em vertical na segunda fase, a impulsão. É na saída que o atleta atinge a maior velocidade angular do quadril, joelhos e tornozelos. Logo em seguida, na fase do voo, o ombro está em $90^{\circ}$ de abdução e estendido, preparando-se para a batida, ou a cortada propriamente dita. Para isso, o ombro estende-se e roda medialmente de maneira brusca e o cotovelo estende-se para $180^{\circ}$. O antebraço prona e o punho flete seguindo o direcionamento que é dado à bola. Após o contato com a bola, o ombro é aduzido, estendido e rodado medialmente, com o cotovelo fletido para prevenir o contato com a rede. A aterrissagem é então realizada com um ou dois pés e o momentum vertical é absorvido pela flexão das articulações dos membros inferiores(2). Assim, ocorre alta demanda de movimentos repetitivos em alta velocidade, muitas vezes em extremos de amplitude de movimento (ADM), que impõem grande estresse mecânico ao complexo do ombro ${ }^{(3)}$.

Lesões no complexo do ombro são comuns em esportes que requerem movimentos repetitivos acima da cabeça e em alta velocidade(4-6). De acordo com a literatura, essa articulação seria a segunda mais acometida por lesões por sobrecarga, variando de 8 a 20\% em atletas de vôlei $i^{(5,7,8)}$. Essas lesões incluem: as tendinopatias do manguito rotador e da porção longa do bíceps braquial e o impacto subacromial. Essas lesões estão frequentemente associadas aos diversos fatores de risco, dentre eles, pouca mobilidade capsular, desequilíbrio muscular, fraqueza, muscular e assimetria do movimento escapular ${ }^{(9)}$.

A musculatura do complexo do ombro possui um papel importante na produção de força e potência articular, permitindo a realização do gesto esportivo de maneira coordenada. Para isso, esses músculos mantêm a estabilidade dinâmica da articulação glenoumeral ${ }^{(10-12)}$. Diversos grupos musculares são considerados importantes para o funcionamento adequado do complexo articular do ombro; entre eles destacam-se os componentes do manguito rotador. Boa capacidade de geração de torque do manguito rotador é essencial para manter a estabilidade e garantir a centralização da cabeça do úmero na cavidade glenoide ${ }^{(13)}$. Kliber $^{(14)}$ descreveu a importância dos pares de força no ombro como sendo mais relevantes do que a força isolada de um músculo na função normal desse complexo articular ${ }^{(7)}$. Além disso, diferença de força entre grupos musculares agonista e antagonista do ombro tem sido apontada como fator de risco importante para lesões nessa articulação(6).

A avaliação isocinética é comumente utilizada para avaliar a condição muscular de atletas saudáveis e lesionados, além de permitir a comparação de diversas variáveis isocinéticas entre atletas e não atletas $^{(15,16)}$. Essa medida de função muscular tem sido proposta como uma ferramenta apropriada para investigar o desempenho muscular em atletas com disfunção da articulação do ombro ${ }^{(4,10)}$. Além disso, os parâmetros isocinéticos podem ser usados como preditores de lesões, principalmente a razão entre rotadores laterais/mediais, uma vez que no voleibol ocorrem movimentos de rotação em alta velocidade, sendo necessário a estabilização dinâmica e equilibrada entre os músculos da articulação glenoumeral $(4,16)$.
A documentação objetiva produzida pela avaliação isocinética auxilia no diagnóstico e na prevenção de lesões do complexo do ombro, além de possibilitar retorno rápido e seguro para a prática esportiva do atleta lesionado ${ }^{(10)}$. Entretanto, os valores de performance muscular avaliados no modo isocinético devem ser considerados como sendo específicos do esporte analisado ${ }^{(17)}$. Dessa forma, dados de outros esportes ou grupo etário não podem ser utilizados como referência para guiar programas de reabilitação ou de prevenção de lesões no voleibol(16,17). Dada a escassez de dados isocinéticos normativos da articulação do ombro em atletas brasileiros de voleibol, o objetivo deste estudo foi avaliar o desempenho muscular isocinético dos músculos rotadores laterais e mediais da articulação glenoumeral em atletas de voleibol da Seleção Brasileira masculina sub-19 e sub-21.

\section{MÉTODOS}

\section{Sujeitos}

Participaram deste estudo 20 atletas da Seleção Brasileira Masculina Sub-19 e 15 da Seleção Brasileira Masculina Sub-21, totalizando 35 atletas de voleibol. Todos os atletas assinaram um termo de consentimento livre e esclarecido para a participação nos testes. Esses testes fizeram parte de um programa de avaliações promovido pelo Centro de Excelência Esportiva (Cenesp-UFMG), no Laboratório de Prevenção e Reabilitação de Lesões Esportivas (Laprev). Os dados descritivos da população estão apresentados na tabela 1.

Tabela 1. Características físicas dos atletas (média \pm desvio padrão)

\begin{tabular}{c|c|c}
\hline & SUB-19 & SUB-21 \\
\hline IDADE (anos) & $17 \pm 0,46$ & $17,19 \pm 1$ \\
\hline MASSA $(\mathrm{kg})$ & $85,6 \pm 9$ & $91,1 \pm 6,6^{*}$ \\
\hline ALTURA $(\mathrm{m})$ & $1,96 \pm 0,05$ & $1,96 \pm 0,06$ \\
\hline${ }^{*} \mathrm{p}<0,05$ &
\end{tabular}

\section{Instrumentação}

O dinamômetro isocinético Biodex 3 System Pro ${ }^{\circledast}$ foi utilizado para a avaliação das variáveis. $O$ teste isocinético se caracteriza pela realização de a velocidade constante e prefixada(17). Esse aparelho aplica uma resistência acomodativa durante toda a amplitude de movimento, ou seja, a resistência do aparelho aumenta de acordo com a força aplicada pelo sujeito avaliado, e não ocorre aumento da velocidade, como acontece em outros exercícios dinâmicos ${ }^{(17)}$.

\section{Procedimentos}

Os atletas foram posicionados na cadeira do dinamômetro com o tronco inclinado em $85^{\circ}$ e estabilizados com cintos no tronco e pélvis para evitar movimentos compensatórios. O posicionamento do membro superior para a avaliação dos rotadores laterais e mediais do ombro foi de: $60^{\circ}$ de abdução do ombro no plano da escápula (30ª̀ frente do plano frontal), $90^{\circ}$ de flexão do cotovelo; o punho foi estabilizado para evitar substituição de movimento. A amplitude de movimento foi limitada a $40^{\circ}$ de rotação lateral e $50^{\circ}$ de rotação medial ( $0^{\circ}$ foi definido com o antebraço na posição horizontal). Essa amplitude foi escolhida para prevenir uma restrição passiva do manguito rotador e o surgimento concomitante de dor, levando a possível alteração nos resultados do teste. Os atletas executaram três repetições submáximas prévias em cada um dos testes nas duas velocidades para fins de familiarização com os procedimentos e para aquecimento prévio.

O protocolo realizado durante o teste foi de cinco repetições máximas de rotação lateral e medial de ombro no modo concêntrico- 
concêntrico na velocidade de $60 \%$ s e 30 repetições na velocidade de $360 \%$ s. Optou-se pela velocidade angular de $60 \%$, pois a produção de força muscular em baixas velocidades leva a maior recrutamento de unidades motoras ${ }^{(18)}$, e assim possibilita melhor representação do trabalho máximo realizado pela musculatura avaliada. Já a velocidade de $360 \%$ s foi selecionada com a intenção de avaliar as variáveis durante velocidade mais funcional para os atletas, dentro do espectro de velocidades permitido pelo dinamômetro. Além disso, o número de repetições realizado nessa velocidade permitiu a avaliação do índice de fadiga dos atletas ${ }^{(17)}$. Durante os testes, os atletas receberam estímulo verbal para garantir desempenho muscular máximo.

\section{Redução dos dados}

As seguintes variáveis isocinéticas foram analisadas: trabalho máximo (joules/kg) e torque máximo (newtons-metros/kg) de rotadores laterais e mediais do ombro normalizados pela massa corporal; razão agonista/antagonista nas velocidades de 60\% e 360\% e índice de fadiga muscular a $360 \%$ s. O trabalho máximo foi calculado como a área total sob a curva torque $x$ ângulo da repetição em que o trabalho calculado foi o maior. Dessa forma, essa variável é mais representativa da função muscular do que a variável torque máximo, pois o trabalho máximo informa a produção de força durante toda a amplitude da contração, enquanto o torque máximo informa o pico de força em apenas um ponto da amplitude total(17). A normalização dos dados para comparações e agrupamentos dos indivíduos foi feita dividindo-se os valores de torque máximo e trabalho máximo pela massa corporal e multiplicados por 100. A variável razão agonista/antagonista foi calculada pela divisão do torque máximo dos músculos rotadores laterais do ombro pelo torque máximo dos músculos rotadores mediais do ombro multiplicados por 100. A variável índice de fadiga foi calculada como a diferença em porcentagem da produção de trabalho entre as dez primeiras e as dez últimas repetições na velocidade de $360 \% \mathrm{~s}^{(17)}$.

\section{Análise estatística}

Para comparar os grupos nas variáveis horas de treinamento por semana e tempo de prática de voleibol, foi utilizado o teste $t$ de Student para amostras independentes. Análises de variância (ANOVA) mistas com um nível fatorial (categorias) e outra de medidas repetidas (membros dominante e não dominante) foram utilizadas para avaliar as variáveis dependentes: trabalho máximo e torque máximo normalizados pela massa corporal dos músculos rotadores laterais e mediais de ombro e para a razão agonista/antagonista nas duas velocidades. Outra ANOVA mista com um nível fatorial (categorias) e outro de medidas repetidas (membros dominante e não dominante) foi utilizada para avaliar o índice de fadiga muscular na velocidade de $360 \%$ s em cada um dos movimentos testados.
Análises de contrastes foram utilizadas para localizar os pares específicos entre os quais a diferença foi significativa. O nível de significância foi estabelecido em $a=0,05$.

\section{RESULTADOS}

Os resultados deste estudo demonstraram diferença estatisticamente significativa ( $p<0,0001$ ) entre as categorias sub-19 e sub-21 para a variável horas de treinamento por semana. No entanto, a variável tempo de prática no voleibol não apresentou diferença entre as categorias $(p>0,52)$. Os valores estão descritos na tabela 2 .

Tabela 2. Características de treinamento (média \pm desvio padrão)

\begin{tabular}{l|c|c|c}
\hline & SUB-19 & SUB-21 & Valor $\boldsymbol{p}$ \\
\hline $\begin{array}{l}\text { Tempo de prática (anos) } \\
\text { Horas de treinamento/ } \\
\text { semana }\end{array}$ & $6 \pm 2,9$ & $5,5 \pm 1,6$ & 0,520965 \\
\hline
\end{tabular}

As ANOVAs revelaram não existir diferenças significativas para as seguintes variáveis: fadiga dos rotadores mediais, torque e trabalho máximo dos rotadores laterais de ombro a $60 \%$ s e torque máximo dos rotadores mediais a 360\% entre categorias, membros e interação membro x categoria (Tabela 3). As análises que apontaram diferenças significativas nos efeitos principais ou interação são apresentadas a seguir:

\section{Torque máximo dos rotadores mediais a $60 \% \mathrm{~s}$}

A análise da variável torque máximo dos rotadores mediais de ombro a $60 \%$ s demonstrou diferenças significativas entre as duas categorias para o membro dominante $(p=0,0001)$ e para membro não dominante $(p=0,0001)$ e ainda entre membros na categoria sub-19 $(p=0,0203)$ e na categoria sub-21 ( $p=0,0292)$ (Tabela 3).

\section{Trabalho máximo dos rotadores mediais a $60 \% / \mathrm{s}$ e $360 \% \mathrm{~s}$}

Para a variável trabalho máximo dos rotadores mediais a $60 \% / \mathrm{s}$ foram encontradas diferenças estatisticamente significativas entre as duas categorias para o membro dominante $(p=0,0001)$ e para membro não dominante $(p=0,0001)$ e também entre membros na categoria sub-19 ( $p=0,0267$ ) e na categoria sub-21 ( $p=0,0166$ ) (Tabela 3).

$\mathrm{Na}$ velocidade de $360 \%$ s, houve diferença estatisticamente significativa para a variável trabalho máximo dos rotadores mediais de ombro entre categorias para o membro dominante $(p=0,0006)$ e para membros não dominantes ( $p=0,0020)$ e entre membros apenas na categoria sub-21 ( $p=0,0473$ ) (Tabela 3).

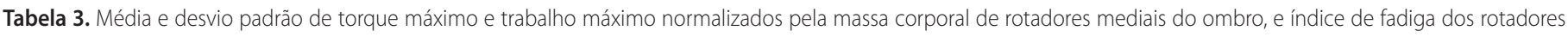
mediais do ombro a $60 \%$ e $360 \%$ s dos membros dominante e não dominante em cada categoria.

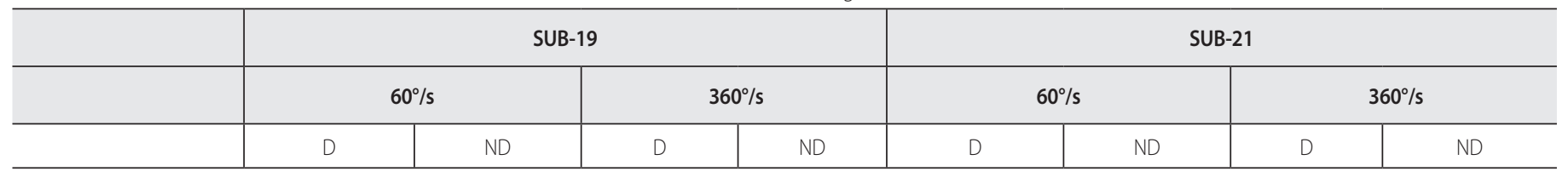

ROTAÇÃO MEDIAL

\begin{tabular}{|c|c|c|c|c|c|c|c|c|}
\hline Torque máximo ( $\mathrm{Nm} / \mathrm{kg})$ & $65,211 \pm 12,970^{a, b}$ & $60,394 \pm 10,172^{a, b}$ & $94,644 \pm 18,173$ & $83,939 \pm 26,132$ & $74,365 \pm 8,995^{a, b}$ & $69,729 \pm 9,192^{a, b}$ & $94,559 \pm 13,549$ & $84,524 \pm 19,264$ \\
\hline Trabalho máximo (J/kg) & $81,0594 \pm 17,965^{a, b}$ & $74,478 \pm 13,931^{a, b}$ & $52,989 \pm 13,143^{\mathrm{a}}$ & $48,622 \pm 9,282^{\mathrm{a}}$ & $104,324 \pm 17,118^{a, b}$ & $96,359 \pm 13,052^{a, b}$ & $63,141 \pm 9,181^{a, c}$ & $57,571 \pm 10,313^{a, c}$ \\
\hline Índice de fadiga (\%) & - & - & $37,978 \pm 14,922$ & $35,189 \pm 9,763$ & - & - & $35,853 \pm 7,718$ & $31,559 \pm 12,751$ \\
\hline
\end{tabular}

$a-p<0,05$ entre as duas categorias para membro dominante e não dominante

b- $p<0,05$ entre membros dominante e não dominante das categorias sub-19 e sub-211

$c-p<0,05$ entre membros dominante e não dominante da categoria sub-21 
Trabalho e torque máximo dos rotadores laterais a $360 \% / \mathrm{s}$

Além disso, a categoria sub-21 apresentou diferença significativa entre membros na variável trabalho máximo dos rotadores laterais do ombro a 360\%/s ( $p=0,0307)$. Em relação à variável torque máximo dos rotadores laterais a 360\% $/ \mathrm{s}$ houve diferença significativa apenas entre as duas categorias para o membro não dominante $(p=0,0308)$ (Tabela 5).

\section{Razão da força muscular entre rotadores laterais e rotadores mediais a $60 \% / \mathrm{s}$ e $360 \% / \mathrm{s}$}

A razão da força muscular entre rotadores laterais e rotadores mediais (RL/RM) a 60\% e 360\% foi estatisticamente significativa entre as categorias, tanto para o membro dominante $(p=0,0016$ e $p=0,0124)$ como para o não dominante $(p=0,0001$ e $p=0,0020)$, respectivamente (Tabela 4).

A variável fadiga dos rotadores laterais do ombro apresentou diferença significativa nos membros não dominantes entre as categorias $(p=0,0260)$ e entre membros nas categorias sub-19 $(p=0,0164)$ e na categoria sub-21 ( $p=0,0073)$ (Tabela 5).

\section{DISCUSSÃO}

Os dados do presente estudo permitem estabelecer valores de referência para força isocinética concêntrica dos rotadores laterais e mediais da articulação glenoumeral em atletas de voleibol masculino das categorias sub-19 e sub-21. O voleibol é um esporte caracterizado pela demanda unilateral do membro superior, o qual acarreta desequilíbrios musculares do complexo do ombro ${ }^{(9)}$. Dessa forma, as diferenças significativas encontradas entre os membros dominante e não dominante nas duas categorias para torque e trabalho máximo dos rotadores internos de ombro a $60 \%$ são, possivelmente, consequência do ganho de força dessa musculatura produzido pelos movimentos de aceleração repetidos durante a cortada. Esses dados corroboram achados de Wang e Cochrane ${ }^{(9)}$, em que os 16 atletas da Seleção Inglesa de Voleibol também apresentaram desequilíbrio entre membros para o torque máximo dos rotadores internos a 60\%s. Entretanto, Gozlan et al.(19) não encontraram diferença significativa entre membros para essa mesma variável em nove atletas profissionais de voleibol. A pequena amostra desse estudo pode ter interferido nos resultados encontrados.
Esportes como o tênis e o beisebol também apresentam movimento do membro superior similar ao do voleibol, levando às mesmas adaptações da musculatura do ombro ${ }^{(20)}$. Assim, a força dos rotadores mediais no membro dominante é maior quando comparada com o não dominante, caracterizando o mesmo desequilíbrio muscular encontrado em jogadores de voleibol ${ }^{(13,20)}$. Esportes que requerem maior simetria entre membros, como a natação, não são caracterizados por esse desequilíbrio muscular, pois esses esportes envolvem treinamento bilateral dos membros superiores, mantendo maior simetria de forças entre membros no complexo do ombro, a qual também é encontrada na população não atleta ${ }^{(19,21)}$

Além da realização de testes concêntricos, vários estudos preconizam o uso da avaliação isocinética excêntrica em atletas, devido à especificidade desse teste em relação ao gesto esportivo, pois na fase de aceleração do braço os rotadores laterais atuam de forma excêntrica ${ }^{(9,10,19,22)}$. Uma limitação do presente estudo foi não realizar o teste isocinético no modo excêntrico. Por outro lado, as dificuldades metodológicas para obtenção de dados isocinéticos excêntricos de qualidade no complexo do ombro fazem com que testes concêntricos ainda sejam considerados como avaliações que fornecem parâmetros importantes sobre o comportamento dos músculos dessa articulação(10).

De acordo com os dados apresentados, a categoria sub-21 treina significativamente mais horas por semana em comparação com a categoria sub-19 (30,3h e 19,9h respectivamente). No esporte de alto rendimento, o atleta aumenta o volume de treinamento de acordo com sua maturação fisiológica e profissional. Sendo assim, o maior valor encontrado para a categoria sub-21 nas variáveis torque máximo a 60\% e trabalho máximo dos rotadores mediais, nas duas velocidades, pode ser justificado pelo maior volume de treinamento. Estudos prospectivos são necessários para entender melhor essa relação entre exposição a treinamentos e desempenho muscular.

Os resultados do presente estudo revelaram que a categoria sub21 apresenta maior desequilíbrio muscular, o qual pode ser explicado pelo maior volume de treinamento. Esse maior volume permite aos atletas sub-21 realizarem maior número de repetição do movimento de rotação medial em relação à categoria sub-19. Além disso, segundo Malliou et al.(21) exercícios gerais de membros superiores na musculação

Tabela 4. Média e desvio padrão de razão agonista/antagonista (laterais/mediais) a $60 \%$ s e $360^{\circ} / \mathrm{s}$ dos membros dominante e não dominante em cada categoria.

\begin{tabular}{|c|c|c|c|c|c|c|c|c|}
\hline & \multicolumn{4}{|c|}{ SUB-21 } & \multicolumn{4}{|c|}{ SUB-19 } \\
\hline & \multicolumn{2}{|c|}{$60^{\circ} / \mathrm{s}$} & \multicolumn{2}{|c|}{$360^{\circ} / \mathrm{s}$} & \multicolumn{2}{|c|}{$60^{\circ} / \mathrm{s}$} & \multicolumn{2}{|c|}{$360^{\circ} / \mathrm{s}$} \\
\hline & $\mathrm{D}$ & ND & $\mathrm{D}$ & ND & $\mathrm{D}$ & ND & $\mathrm{D}$ & ND \\
\hline RAZÃO AG/ANT (\%) & $74,011 \pm 11,750^{a}$ & $78,950 \pm 15,318^{b}$ & $79,722 \pm 24,075^{a}$ & $88,528 \pm 21,835^{b}$ & $62,765 \pm 12,050 a$ & $64,235 \pm 7,302^{b}$ & $64,765 \pm 21,282^{a}$ & $69,588 \pm 3,811^{b}$ \\
\hline
\end{tabular}

a- $p<0,05$ entre as duas categorias para membros dominantes.

$\mathrm{b}-\mathrm{p}<0,05$ entre as duas categorias para membros näo dominantes.

Tabela 5. Média e desvio padrão de torque máximo e trabalho máximo normalizados pela massa corporal de rotadores laterais do ombro, índice de fadiga de rotadores laterais do ombro a $60 \% \mathrm{~s}$ e $300 \% \mathrm{~s}$ dos membros dominante e não dominante em cada categoria.

\begin{tabular}{c|c|c|c|c|c|c|c|c}
\hline & \multicolumn{3}{|c|}{ SUB-21 } & \multicolumn{3}{c}{ SUB-19 } \\
\hline & \multicolumn{2}{|c|}{$60^{\circ} / \mathrm{s}$} & \multicolumn{2}{|c|}{$360^{\circ} / \mathrm{s}$} & \multicolumn{2}{c}{$60^{\circ} / \mathrm{s}$} & $360^{\circ} / \mathrm{s}$ \\
\hline & $\mathrm{D}$ & $\mathrm{ND}$ & $\mathrm{D}$ & $\mathrm{ND}$ & $\mathrm{D}$ & $\mathrm{ND}$ & $\mathrm{D}$ & $\mathrm{ND}$ \\
\hline
\end{tabular}

\section{ROTAÇÃO LATERAL}

\begin{tabular}{|c|c|c|c|c|c|c|c|c|}
\hline Torque máximo $(\mathrm{Nm} / \mathrm{kg})$ & $47,494 \pm 5,173$ & $45,989 \pm 6,441$ & $74,289 \pm 22,317$ & $72,056 \pm 25,112^{\mathrm{a}}$ & $45,982 \pm 7,601$ & $44,812 \pm 3,205$ & $63,871 \pm 18,760$ & $57,441 \pm 18,708^{a}$ \\
\hline Trabalho máximo (J/kg) & $59,778 \pm 7,734$ & $57,544 \pm 8,017$ & $32,928 \pm 9,198$ & $31,228 \pm 7,156$ & $56,771 \pm 10,546$ & $57,018 \pm 5,723$ & $32,318 \pm 7,613^{b}$ & $28,088 \pm 4,709^{b}$ \\
\hline Índice de fadiga (\%) & - & - & $35,183 \pm 8,908^{c}$ & $46,733 \pm 13,660^{a, c}$ & - & - & $44,106 \pm 16,552^{b}$ & $57,535 \pm 14,599^{b}$ \\
\hline
\end{tabular}

$a-p<0,05$ entre as duas categorias para o membro não dominante

$b-p<0,05$ entre membros dominante e não dominante da categoria sub-21.

$c-p<0,05$ entre membros dominante e não dominante da categoria sub-19. 
(pulley, remada, supino) produzem aumento da força nos músculos rotadores mediais e não dos laterais. Possivelmente, esse fator contribuiu para o maior desequilíbrio na categoria sub-21, uma vez que esses atletas possuem mais tempo de prática na musculação com maior intensidade. Nesse sentido, os profissionais responsáveis pela preparação física (preparador físico e fisioterapeuta) devem considerar esse fator ao montar o programa de musculação desses atletas, para minimizar possíveis efeitos que levem a desequilíbrios musculares e/ou modificações posturais, como anteriorização da cabeça umeral, inclinação anterior da escápula e rotação interna da escápula, que predispõem à síndrome do impacto e a sintomas articulares ${ }^{(9)}$, intervindo assim de modo preventivo.

Na literatura, existe uma variação dos valores de referência para razão RL/RM da articulação do ombro(22). Segundo Ellenbecker e Mattalino(23), para indivíduos não atletas e sem queixas na articulação do ombro, a razão RL/RM fica em torno de $66 \%$ a $60 \%$ s; esse mesmo valor foi encontrado por Chandler et al.(20) para atletas de tênis. Codine et al. ${ }^{(13)}$ encontraram valores para não atletas de $70 \%$ na velocidade de $60 \%$ no membro dominante e $75 \%$ no membro não dominante e, na velocidade de $300 \%$, encontraram $75 \%$ para o membro dominante e $81 \%$ para o não dominante. Além disso, esses autores compararam os dados de não atletas com os de atletas de diversas modalidades como: corredores (78\% membro dominante e $83 \%$ para o não dominante a $60 \%$ s e $75 \%$ no dominante e $84 \%$ no não dominante a $300 \%$ s), tenistas (68\% para dominante e $74 \%$ para não dominante a $60 \%$ s e $67 \%$ no dominante e $76 \%$ no não dominante a $300 \%$ s) e jogadores de beisebol (61\% para o membro dominante e $71 \%$ para o não dominante a $60 \%$ e $57 \%$ no dominante e $65 \%$ no não dominante na velocidade de $300 \%$ s) ${ }^{(13)}$. Sendo assim, os não atletas e corredores tiveram as maiores razões, seguidos dos atletas de tênis e beisebol. No presente estudo, apenas os atletas brasileiros de voleibol da categoria sub-21 apresentaram valores abaixo dos de referência para razão RL/RM da articulação do ombro, caracterizando déficit de força dos rotadores laterais e/ou

\section{REFERÊNCIAS}

1. Forthomme B, Croisier JL, Ciccarone G, Crielaard JM, Cloes M. Factors correlated with volleyball spike velocity. Am J Sports Med 2005;33:1513-9.

2. Coleman SGS, Benham AS, Northcott SR. A three-dimensional cinematographical analysis of the volleyball spike. J Sports Sci 1993;11:295-302.

3. Witrouw E, Cools A, Lysens R, Cambier D, Vanderstraeten G, Victor J, et al. Suprascapular neuropathy in volleyball players. Br J Sports Med 2005;34:174-80.

4. Noffal GJ. Isokinetic eccentric-to-concentric strength ratios of the shoulder rotator muscles in throwers and nonthrowers. Am J Sports Med 2003;31:537-41

5. Briner WW, Benjamin HJ. Volleyball injuries: managing acute and overuse disorders. Phys Sportsmed 1999;27:48-60..

6. Gabriel YF, Patrick CW. A study of antagonist/agonist isokinetic work ratios of shoulder rotators in men who play badminton. J Ortop Sports Phys Ther 2002;32:399-404.

7. Solgard L, Nielsen AB, Moller-Madsen B, Jacobsen BW, Yde J, Jensen J. Volleyball injuries presenting in casualty: a prospective study. Br J Sports Med 1995;29:200-4.

8. Bahr R, Reeser JC. Injuries among world-class professional beach volleyball players. Am J Sports Med 2003;31:119-25.

9. Wang $\mathrm{H}$, Cochrane T. Mobility impairment, muscle imbalance, muscle weakness, scapular asymmetry and shoulder injury in elite volleyball athletes. J Sports Med Phys Fitness 2001;41:403-10.

10. Ellenbecker TS, Davies GJ. The application of isokinetics in testing and rehabilitation of the shoulder complex. J Athl Train 2000;35:338-50.

11. Wilk KE, Andrews JR, Arrigo CA, Keirns MA, Erber DJ. The strength characteristics of internal and external rotator muscles in professional baseball pitchers. Am J Sports Med 1993;21:61-6.

12. Alderink GJ, Kuck DJ. Isokinetic shoulder strength of high school and college-aged pitchers. J Orthop Sports Phys Ther 1986;7:163-72. aumento de força dos rotadores mediais. Isso pode decorrer da especificidade do gesto esportivo, associado a maior quantidade de horas de treinamento dessa categoria, como discutido no parágrafo anterior.

A variável fadiga dos rotadores laterais do ombro apresentou diferença estatisticamente significativa entre as categorias para os membros não dominantes e entre membros nas categorias sub-19 e na sub-21. Em ambas, o lado dominante obteve menor média nessa variável, ou seja, esse teria maior predisposição à fadiga muscular. Dessa forma, faz-se necessário um trabalho especifico de resistência dos rotadores laterais, pois esse grupo muscular, por atuar excentricamente no movimento da cortada, pode estar sujeito a fadiga mais precoce, principalmente quando considerados os desequilíbrios musculares do complexo do ombro, os quais podem interferir na performance ou predispor à lesão(9,10).

A partir dos resultados deste estudo é possível o estabelecimento de critérios objetivos para a implementação de um programa de prevenção direcionado ao desempenho muscular para atletas de voleibol. Os dados apresentados permitiram a identificação de algumas alterações em atletas de voleibol que, associadas a demandas específicas do esporte, podem levar à sintomatologia no complexo do ombro e/ ou queda da performance.

\section{CONCLUSÃO}

Os dados apresentados demonstraram haver um desequilíbrio entre os rotadores laterais e mediais da articulação do ombro em atletas brasileiros de voleibol da categoria sub-21. Foi constatado ainda que esses atletas possuem maior volume de treinamento e maior desempenho de rotadores mediais do ombro quando comparados com a categoria sub-19. Além disso, ambas as categorias apresentaram fadiga somente dos músculos rotadores laterais.

Todos os autores declararam não haver qualquer potencial conflito de interesses referente a este artigo.
13. Codine P, Bernard PL, Pocholle M, Benaim C, Brun V. Influence of sports discipline on shoulder rotator cuff balance. Med Sci Sports Exerc 1997;29:1400-5.

14. Kliber WB. The role of scapular in athletic shoulder function. Am J Sports Med 1998;22:325-37.

15. Baltaci G, Tunay VB. Isokinetic performance at diagonal pattern and shoulder mobility in overhead athletes. Scand J Med Sci Sports 2004;14:231-8.

16. Hughes RE, Johnson ME, O'Driscoll SW, An KN. Normative values of agonist-antagonist shoulder strength ratios of adults aged 20 to 78 years. Arch Phys Med Rehabil 1999;80:1324-6.

17. Wilk KE. Isokinetic Testing: goals, standards and knee test interpretation. In: Biodex Medical System. Biodex System 3. Advantage Software. Operations Manual.

18. Siqueira CM, Pelegrini FR, Fontana MF, Greve JM. Isokinetic dynamometry of knee flexors and extensors: comparative study among non-athletes, jumper athletes and runner athletes. Rev Hosp Clin Fac Med São Paulo 2002;57:19-24.

19. Gozlan G, Bensoussan L, Coudreuse J-M, Fondarai J, Gremeaux V, Viton JM, et al. Isokinetic dynamometry measurement of shoulder rotation strength in healthy elite athletes (swimming, volleyball, tennis): comparision between dominant and nondominant shoulder. Ann Readapt Med Phys 2006;49:8-15.

20. Chandler TJ, Kibler WB, Stracener EC, Ziegler AK, Pace B. Shoulder strength, power, and endurance in college tennis players. Am J Sports Med 1992;20:455-8.

21. Malliou PC, Giannakopoulos K, Beneka AG, Gioftsidou A, Godolias G. Effective ways of restoring muscular imbalances of various training methods the rotator cuff muscle group: a comparative study. $\mathrm{Br}$ J Sports Med 2004;38:766-72.

22. Yildiz Y, Aydin T, Sekir U, Kiralp MZ, Hazneci B, Kalyon TA. Shoulder terminal range eccentric antagonist/ concentric agonist strength ratios in overhead athletes. Scand J Med Sci Sports 2006;16:174-80.

23. Ellenbecker TS, Mattalino AJ. Concentric isokinetic shoulder internal and external rotation strength in professional baseball pitchers. J Orthop Sports Phys Ther 1997;25:323-8. 